\title{
Measurement of positronium hyperfine splitting with quantum oscillation
}

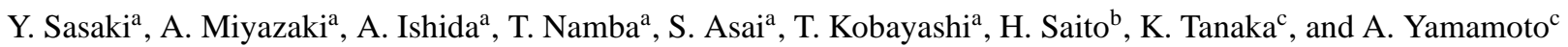 \\ ${ }^{a}$ Department of Physics, Graduate School of Science, and International Center for Elementary Particle Physics, \\ The University of Tokyo, 7-3-1 Hongo, Bunkyo-ku, Tokyo, 113-0033, Japan \\ ${ }^{b}$ Institute of Physics, Graduate School of Arts and Sciences, The University of Tokyo, 3-8-1 Komaba, Meguro-ku, Tokyo, 153-8902, Japan \\ ${ }^{c}$ High Energy Accelerator Research Organization (KEK), 1-1 Oho, Tsukuba, Ibaraki, 305-0801, Japan
}

\section{Abstract}

Interference between different energy eigenstates in a quantum system results in an oscillation with a frequency which is proportional to the difference in energy between the states. Such an oscillation is observable in polarized positronium when it is placed in a magnetic field. In order to measure the hyperfine splitting of positronium, we perform the precise measurement of this oscillation using a high quality superconducting magnet and fast photon-detectors. A result of $203.324 \pm 0.039$ (stat.) \pm 0.015 ( sys.) GHz is obtained which is consistent with both theoretical calculations and previous precise measurements.

'Keywords: QED, positronium, quantum oscillation

\section{Introduction}

Positronium (Ps), the bound state of an electron and a positron, is the lightest hydrogen-like atom. Since it is a purely leptonic system and is thus free from the uncertainties of hadronic interactions, it is an excellent object for studying Quantum Electrodynamics (QED), especially for the bound state. The two ground states of positronium, the triplet state $\left(1^{3} S_{1}\right)$ and the singlet state $\left(1^{1} S_{0}\right)$, are known as orthopositronium (o-Ps) and para-positronium ( $p$-Ps), respectively. The difference in the energy between $o$-Ps and p-Ps is called hyperfine splitting (HFS), whose value is about $203 \mathrm{GHz}$, which is significantly larger than that for the hydrogen atom $(1.4 \mathrm{GHz})$. 'A theoretical prediction including $O\left(\alpha^{3}\right)$ corrections has been recently obtained using a Non-Relativistic Quantum Electrodynamics (NRQED) approach [1]. The result of this calculation deviates from the previously measured values [2, 3] by a significant margin $(3.9 \sigma, 15 \mathrm{ppm})$. This discrepancy might indicate the signal of new physics beyond the Standard Model similar to the discrepancy in the measurement of the muon anomalous magnetic moment [4]. Both experimental results and the QED prediction should be examined again to confirm the discrepancy 1.

In a static magnetic field, the two states $\left|s=1, m_{z}=0\right\rangle$ and $\left|s=0, m_{z}=0\right\rangle$ mix to the states $|+\rangle$ and $|-\rangle[7]$ :

$$
\begin{aligned}
& |+\rangle=C_{1}^{1}\left|s=1, m_{z}=0\right\rangle+C_{0}^{1}\left|s=0, m_{z}=0\right\rangle, \\
& |-\rangle=C_{1}^{0}\left|s=1, m_{z}=0\right\rangle+C_{0}^{0}\left|s=0, m_{z}=0\right\rangle,
\end{aligned}
$$

and

$$
C_{1}^{0}=-C_{0}^{1}=\left\{\frac{1}{2}\left[1-\left(1+\chi^{2}\right)^{-\frac{1}{2}}\right]\right\}^{\frac{1}{2}},
$$

\footnotetext{
${ }^{1}$ In the previous experimental results, there may be a systematic error in the material effect due to non-thermalized positronium [5] like the $o$-Ps lifetime puzzle [6].
}

$$
C_{1}^{1}=C_{0}^{0}=\left\{\frac{1}{2}\left[1+\left(1+\chi^{2}\right)^{-\frac{1}{2}}\right]\right\}^{\frac{1}{2}},
$$

where $\chi=\frac{2 g^{\prime} \mu_{B} H}{h \Delta_{\mathrm{HFS}}}, \Delta_{\mathrm{HFS}}$ is the HFS value, $H$ is the static magnetic field strength, $\mu_{B}$ is the Bohr magneton, $h$ is the planck constant, and $g^{\prime}=g\left(1-\frac{5}{24} \alpha^{2}\right)$ is the $g$-factor of the electron (positron) including the bound state correction [8].

The other states $\left|s=1, m_{z}= \pm 1\right\rangle$ do not couple with the static magnetic field, and thus remain unperturbed. The energy splitting between $|+\rangle$ and $\left|s=1, m_{z}= \pm 1\right\rangle$ (the Zeeman splitting) is

$$
\Delta_{\text {mix }}=\frac{\Omega}{2 \pi}=\frac{\Delta_{\mathrm{HFS}}}{2}\left(\sqrt{1+\chi^{2}}-1\right) .
$$

All previous experiments obtained the value of the HFS via the formula above by measuring the Zeeman splitting in a magnetic field of a known strength [9]. There are two distinct approaches for measuring the Zeeman splitting. The first approach, which was proposed in Ref. [10], uses an external high power light source with a resonant frequency of $\Delta_{\text {mix }}$ (about $3 \mathrm{GHz}$ in a magnetic field of $\approx 0.8 \mathrm{~T}$ ) to stimulate the transition from $\left|s=1, m_{z}= \pm 1\right\rangle$ to $|+\rangle$. In this case, the stability of the power, frequency of the light source, and the quality of the RF cavity are crucial. This approach has been used in many previous experiments, for example Mills et al. [2] and Ritter et al. [3], and has resulted in measurements with accuracies of $O(1) \mathrm{ppm}$.

The second approach proposed by V. G. Baryshevsky et al. [7] makes use of the quantum oscillation between $\mid s=$ $\left.1, m_{z}= \pm 1\right\rangle$ and $|+\rangle$. Positrons emitted from a $\beta^{+}$source are polarized in the direction of their momentum due to parity violation in the weak interaction 2 . Consequently, the re-

\footnotetext{
${ }^{2}$ The polarization ratio $P$ is determined by the initial velocity $v$ of the positron, $P=v / c$.
} 
sulting $o$-Ps is also highly polarized. This $o$-Ps is in a superimposed state of $|+\rangle$ and $\left|s=1, m_{s}= \pm 1\right\rangle$, and thus oscillates with a frequency $\Omega / 2 \pi$. This oscillation was observed by Baryshevsky et al. in a subsequent experiment [11]. This approach is free from systematic errors due to the high power light source and the RF cavity with a high Q-value. Instead, a high-performance time-to-digital converter (TDC) is crucial for precise measurement of the time spectrum. Those two complementary approaches are necessary to understand the discrepancy in the theoretical prediction and the experimental values of the HFS. In 1996, S. Fan et al. [12] performed an improved experiment using this quantum oscillation method, obtaining a result of $202.5 \pm 3.5 \mathrm{GHz}$.

In this paper, we greatly improve the accuracy of the measurement with the quantum oscillation method by using a very high quality magnetic field, a fast photon-detection system, and high quality TDCs. This method is based on the spin rotation of $o$-Ps (Ps-SR). It is interesting to note that, using the relaxation of $o$-Ps spin, Ps-SR can be used for probing various materials in material science research [13]. Since positronium is much lighter than a muon, the relaxation processes of positronium spin are expected to be much more sensitive to local magnetic structures than $\mu$-SR 3 .

\section{Experimental setup}

The top panel of Fig. 1 shows a schematic diagram of the experimental setup, while the bottom panel shows a magnified view of the positronium chamber. $\mathrm{A}{ }^{68} \mathrm{Ge}-\mathrm{Ga} \beta^{+}$source, whose end point energy is $1.9 \mathrm{MeV}$, is used. The radioactivity is $30 \mathrm{kBq}$, and is distributed in the active diameter of $9.35 \mathrm{~mm}$. A positron passes through a plastic scintillator (NE102) with a thickness of $500 \mu \mathrm{m}$. The resulting two light pulses are transmitted in both directions by light guides to two photomultipliers (PMT; Hamamatsu R5924-70). The positron then stops and forms positronium in the silica aerogel target, which is $10 \mathrm{~mm}$ in diameter and $10 \mathrm{~mm}$ long, with a density of $0.11 \mathrm{~g} / \mathrm{cm}^{3}$. The surface of the primary grain is made hydrophobic in order to avoid the Stark effect from the electric dipole of the hydroxyl groups. The plastic scintillator tags the positron emitted along the direction of the $x$-axis, which results in polarized $o$-Ps along the $x$-axis. The polarization ratio is estimated to be 0.23 by Geant4 simulation, in which the geometry, the threshold of the plastic scintillator and the velocity distribution of positrons are considered. The entire positronium system is contained within a chamber evacuated with a rotary pump in order to reduce pickoff annihilation [14].

The magnetic field (z-direction) is provided by a superconducting magnet which was originally developed for medical NMR use. It has a large bore diameter of $80 \mathrm{~cm}$ and an excellent uniformity of $10 \mathrm{ppm}$ over the volume of the silica aerogel. The magnetic field is measured with an NMR magnetometer (ECHO-ELECTRONICS, EFM-150HM-AX) which has a calibration uncertainty of $35 \mathrm{ppm}$.

\footnotetext{
${ }^{3}$ Unfortunately, the lifetime of $o$-Ps is much shorter than that of a muon.
}

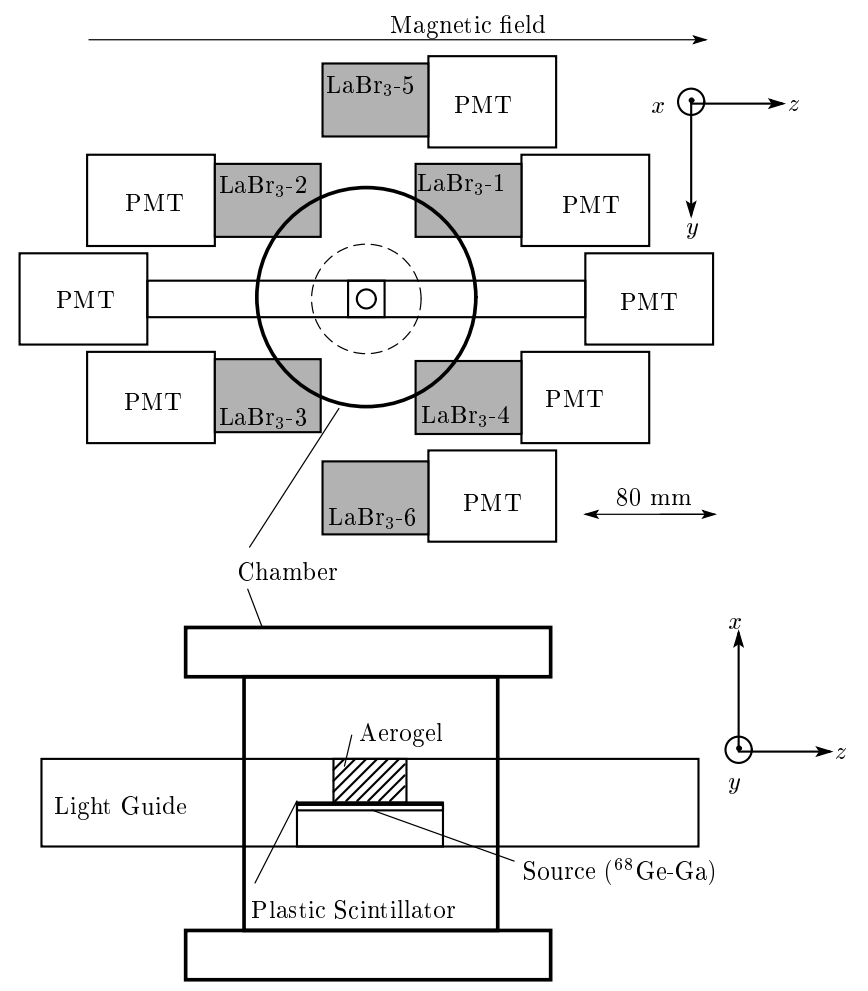

Figure 1: Schematic diagram of the experimental setup. The top panel shows the entire experimental setup. The magnetic field direction is along the $z$-axis, while the $\mathrm{LaBr}_{3}(\mathrm{Ce})$ scintillators are placed in the $y z$-plane. The direction of the $\beta^{+}$emitted from the ${ }^{68} \mathrm{Ge}-\mathrm{Ga}$ source is along the $x$-axis. The bold circle is the positronium chamber. The bottom panel is a magnified view of the positronium chamber, in which the ${ }^{68} \mathrm{Ge}-\mathrm{Ga}$ source, the thin plastic scintillator, and the silica aerogel are located. The coordinate systems are separately defined in the two panels.

The produced $o$-Ps decays into three gamma rays, which are then detected by six $\mathrm{LaBr}_{3}$ crystals of 1.5 inches in diameter and 2 inches long. PMTs (Hamamatsu R5924-70) are attached to these crystals. The $\mathrm{LaBr}_{3}$ detectors are located at $(\theta, \phi)=\left(\frac{\pi}{4},-\frac{\pi}{2}\right),\left(\frac{3 \pi}{4},-\frac{\pi}{2}\right),\left(\frac{3 \pi}{4}, \frac{\pi}{2}\right),\left(\frac{\pi}{4}, \frac{\pi}{2}\right),\left(\frac{\pi}{2},-\frac{\pi}{2}\right),\left(\frac{\pi}{2}, \frac{\pi}{2}\right)$, where $\theta=\arccos \left(\frac{z}{\sqrt{x^{2}+y^{2}}}\right)$ and $\phi=\arctan \left(\frac{y}{x}\right)$. The detectors are referred to as $\mathrm{LaBr}_{3}-1$ to $\mathrm{LaBr}_{3}-6$ (see Fig. 1). The quantum oscillation modulates the angular distribution of the three gamma rays emitted from the $o$-Ps decay, which leads to beats in the decay curve of $o$-Ps. Unlike the muon precession, in which the emission direction of $\mu \rightarrow e$ rotates, this oscillation changes its angular distribution as a vibration in the $y z$-plane. This is a unique property of a spin-1 system. The $\mathrm{LaBr}_{3}-1$ and $\mathrm{LaBr}_{3}-3$ detector pair observes the oscillation with the same phase, while the $\mathrm{LaBr}_{3}-2$ and $\mathrm{LaBr}_{3}-4$ detector pair observes the inverse phase. The $\mathrm{LaBr}_{3}-5$ and $\mathrm{LaBr}_{3}-6$ detectors observe the exponential decay curve without the oscillation.

Fig. 2 shows the energy spectrum measured with one of the $\mathrm{LaBr}_{3}$ crystals. We note that a good energy resolution of $4.0 \%(\mathrm{FWHM})$ at $511 \mathrm{keV}$ is obtained. The time resolutions of the $\mathrm{LaBr}_{3}$ detectors for the $511 \mathrm{keV}$ gamma peak and the positron tagging plastic scintillator are $200 \mathrm{ps}$ (FWHM) and $3.8 \mathrm{~ns}$ (FWHM), respectively. These values are obtained with 


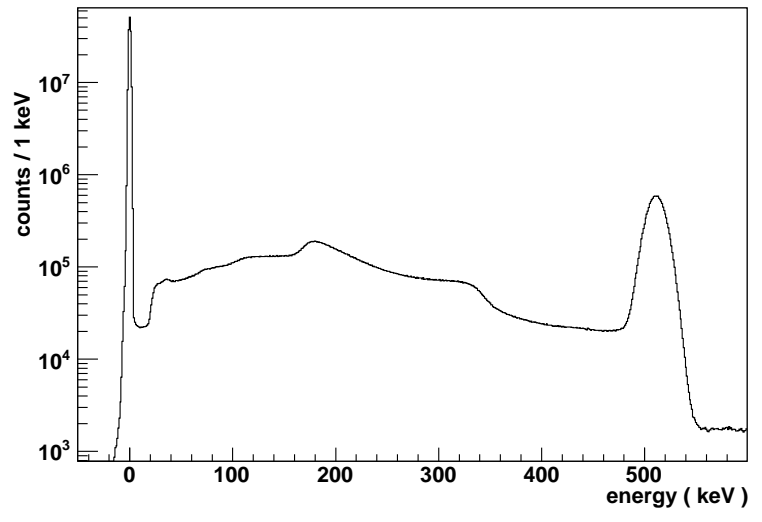

Figure 2: Energy spectrum of a $\mathrm{LaBr}_{3}$ crystal measured in the magnetic field of $100 \mathrm{mT}$.

the PMTs located in a magnetic field of $100 \mathrm{mT}$.

Data acquisition starts with the rate of $740 \mathrm{~Hz}$ when the plastic scintillator signal is coincident within $-50 \mathrm{~ns}$ to $1650 \mathrm{~ns}$ with at least one of the $\mathrm{LaBr}_{3}$ signals. The time $t=0$ is defined as the timing of the plastic scintillator pulse. A charge ADC (CAEN $\mathrm{C} 1205)$ is used to measure the energy information of the $\mathrm{LaBr}_{3}$ crystals, while another charge ADC (REPIC RPC-022) is used to measure both the baseline information of the $\mathrm{LaBr}_{3}$ crystals and the energy information of the plastic scintillator. The baseline information is measured just before the gamma ray arrives at the $\mathrm{LaBr}_{3}$ crystals in order to remove pile-up events. The time differences between the plastic scintillator and $\mathrm{LaBr}_{3}$ scintillators are measured by direct clock TDCs $(5 \mathrm{GHz}$ : time resolution of $200 \mathrm{ps}$ ). These TDCs have excellent integral and differential linearities.

Separate measurements have been made for 5 different magnetic field strengths: $0 \mathrm{mT}, 100 \mathrm{mT}, 118 \mathrm{mT}, 135 \mathrm{mT}$ and $138 \mathrm{mT}$. Also, both $+x$ (up) and $-x$ (down) polarization measurements were performed by turning the positronium chamber upside down. The expected time periods of the oscillation are about $26 \mathrm{~ns}$ and $14 \mathrm{~ns}$ for $100 \mathrm{mT}$ and $138 \mathrm{mT}$, respectively. The period of each run was about 3 days. In total, $1.4 \times 10^{9}$ events were recorded in about 22 days of data acquisition period. The energy and timing spectra are calibrated every one hour using the $511 \mathrm{keV}$ and pedestal peaks.

\section{Analysis}

The following event selections are applied in order to obtain a clean time spectrum:

1. In order to remove pile-up events, the fluctuation of the baseline of the $\mathrm{LaBr}_{3}$ is required to be smaller than $3 \sigma$ (where $\sigma$ is the noise level).

2. The events in which more than two $\mathrm{LaBr}_{3}$ crystals are hit simultaneously are disregarded. This helps to reduce the accidental contribution since accidental events have a back-to-back topology.

3. In order to obtain a good time resolution, the energy deposited in the $\mathrm{LaBr}_{3}$ is required to be larger than $100 \mathrm{keV}$.
Table 1: An example of the fitted result using the separate fitting method for $100 \mathrm{mT}$ (up).

\begin{tabular}{cc} 
Parameter & Fitted Value \\
\hline$A_{1}$ & $0.095 \pm 0.011$ \\
$B_{1}$ & $0.078 \pm 0.012$ \\
$C_{1}$ & $0.0096 \pm 0.0003$ \\
$D_{1}$ & $0.00733 \pm 0.00001$ \\
$\theta_{1}(\mathrm{rad})$ & $0.18 \pm 0.03$ \\
\hline$\gamma_{1}\left(\mathrm{~ns}^{-1}\right)$ & $0.00733 \pm 0.00012$ \\
$\gamma_{2}\left(\mathrm{~ns}^{-1}\right)$ & $0.00975 \pm 0.00024$ \\
$\Omega(\mathrm{rad} / \mathrm{ns})$ & $0.24573 \pm 0.00017$ \\
\hline$\chi^{2} / \mathrm{ndf}$ & $1.00(\mathrm{ndf}=8370)$ \\
\hline
\end{tabular}

Since the multiple hit events are removed, six statistically independent time spectra are obtained. We then fit the spectra using two distinct methods: the separate fitting method and the subtracting method.

\subsection{Separate fitting method}

Fig. 3 shows the timing spectra with the best fitted result using the separate fitting method. In this method, the six time spectra are simultaneously fitted in the range $50 \mathrm{~ns}$ to $1450 \mathrm{~ns}$ with the following functions:

$$
\begin{aligned}
f_{n}(t)= & A_{n} e^{-\gamma_{1} t}+B_{n} e^{-\gamma_{2} t} \\
+ & C_{n} e^{-\frac{\gamma_{1}+\gamma_{2}}{2} t} \times \sin \left(\Omega t+\theta_{n}\right) \\
+ & D_{n} \\
& \text { for } n=1,2,3,4 \\
f_{n}(t)= & A_{n} e^{-\gamma_{1} t}+B_{n} e^{-\gamma_{2} t} \\
+ & D_{n} \\
& \text { for } n=5,6
\end{aligned}
$$

where $A$ and $B$ are proportionality constants for the decay curves, $C$ is the oscillation amplitude, and $D$ is a constant for accidental hits ( $n$ denotes the index of the $\mathrm{LaBr}_{3}$ detector). The two decay rates $\gamma_{1}$ and $\gamma_{2}$, and the angular frequency of the oscillation $\Omega$ are common variables among the six functions, while the others are kept free. $\gamma_{1}$ and $\gamma_{2}$ are decay rates for $\left|s=1, m_{z}= \pm 1\right\rangle$ and $|+\rangle$, respectively. These rates include the effect of pick-off annihilation. $\Omega$ is the Zeeman splitting frequency defined in Eq. (5).

The results of the fits for $\mathrm{LaBr}_{3}-1$ are listed in Table 1 for the $100 \mathrm{mT}$ case. All fitted variables converged as shown in the table, in which a reasonable $\chi^{2} /$ ndf of 1.00 is obtained. The fitted lifetime values $1 / \gamma_{1}, 1 / \gamma_{2}$ are $136.4 \pm 2.2 \mathrm{~ns}$ and $102.5 \pm 2.5 \mathrm{~ns}$, which are consistent with the lifetime $\left(\left|s=1, m_{z}= \pm 1\right\rangle\right)$ measured in aerogel [14] and the calculated value $(|+\rangle)$ in a magnetic field of $100 \mathrm{mT}$, respectively. A fitted time period $2 \pi / \Omega$ of $25.57 \pm 0.02 \mathrm{~ns}(700 \mathrm{ppm})$ is obtained for this run.

The same procedure is applied for the runs with different magnetic field strengths and different polarizations. The resulting $\chi^{2} /$ ndf values are always found to be less than 1.03. $\Delta_{\mathrm{HFS}}$ can be calculated from the fitted $\Omega$.The obtained $\Delta_{\text {HFS }}$ are listed in Table 2 for the various magnetic field strengths. 

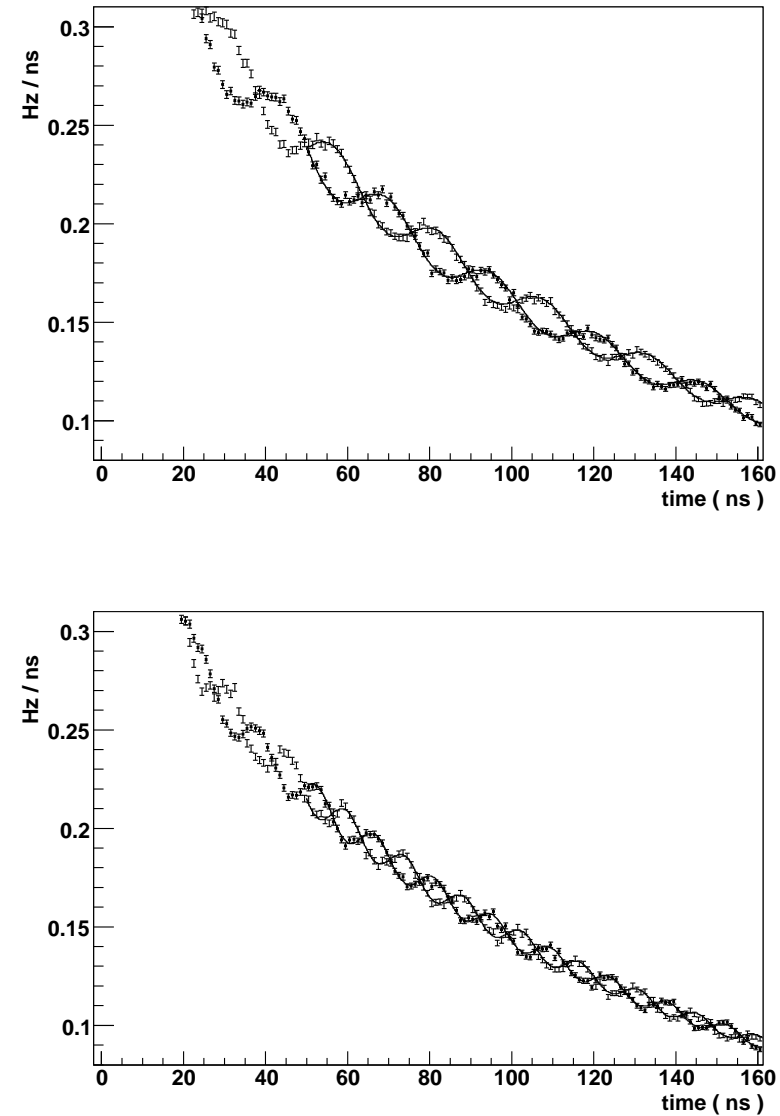

Figure 3: The timing spectra obtained in the separate fitting method for $100 \mathrm{mT}$ (top) and $135 \mathrm{mT}$ (bottom). In both panels, the data points are plotted with error bars, while the solid lines show the best fitted results. The opposite phase spectra are superimposed in both panels. The polarization direction of $\beta^{+}$ is upwards.

The $118 \mathrm{mT}$ (up) measurement is also performed using a different TDC clock of $8 \mathrm{GHz}$, in which consistent results are obtained. This is an important check for the TDC, since the time spectrum is crucial for this experiment.

The $\Delta_{\text {HFS }}$ values obtained at the various magnetic field strengths are consistent with each other. The combined value is $203.336 \pm 0.048$ (stat.) $\mathrm{GHz}$.

\subsection{Subtracting method}

In this method, the sum of the $\mathrm{LaBr}_{3}-2$ and $\mathrm{LaBr}_{3}-4$ spectra are subtracted from the sum of the $\mathrm{LaBr}_{3}-1$ and $\mathrm{LaBr}_{3}-3$ spectra. Ideally, this would cancel the exponential components in the spectra, leaving only the oscillating component. However, the acceptances of the $\mathrm{LaBr}_{3}$ detectors are not exactly the same, which results in small exponential components remaining after the subtraction. Although these components are thus still included in the fits, the upshot is that the oscillating component is greatly enhanced. Furthermore, the cancellation of the prompt peak means that the fitting region can be extended closer to zero. Therefore, this method has a smaller statistical error in the fits. Fig. 4 shows examples of the subtracted time
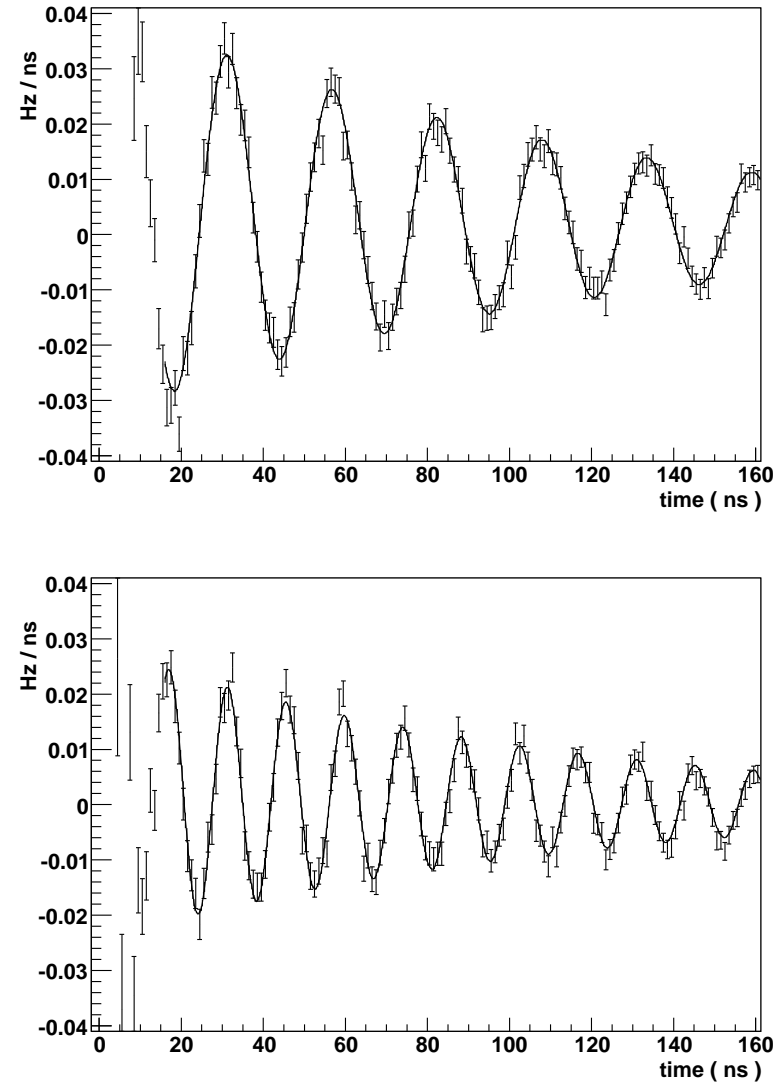

Figure 4: Time spectra obtained in the subtracting method for $100 \mathrm{mT}$ (top) and $135 \mathrm{mT}$ (bottom). In both panels, the data points are plotted with error bars, while the solid lines show the best fitted results.

spectra with the best fits superimposed. The fitting region is set from $16 \mathrm{~ns}$ to $1416 \mathrm{~ns}$. The fitting function is given by

$$
\begin{aligned}
f(t) & =A e^{-\gamma_{1} t}+B e^{-\gamma_{2} t} \\
& +C e^{-\frac{\gamma_{1}+\gamma_{2}}{2} t} \times \sin (\Omega t+\theta) \\
& +D
\end{aligned}
$$

where $\gamma_{1}, \gamma_{2}$, and $\Omega$ are the same as the ones in the separate fitting method. The two exponential components with proportionality constants $A$ and $B$ are for the remnant decay curves, while the component with constant $C$ is the oscillation contribution. The amplitudes $A, B$ and $D$ are expected to be small.

The fitted results are listed in Table 3 for the $100 \mathrm{mT}$ case. The coefficients $A$ and $B$ are consistent with zero and $D$ is also much smaller than $C$, which means that the cancellation works well. A fitted time period $2 \pi / \Omega$ of $25.59 \pm 0.01 \mathrm{~ns}(590 \mathrm{ppm})$ is obtained for this run.

The obtained $\Delta_{\mathrm{HFS}}$ are listed in Table 4 for the various magnetic field strengths. The $\Delta_{\text {HFS }}$ values obtained at the various magnetic field strengths are consistent with each other. The combined value is $203.324 \pm 0.039$ (stat.) $\mathrm{GHz}$. 
Table 2: Summary of the HFS values obtained using the separate fitting method. (up) and (down) denote the direction of the $\beta^{+}$with respect to the $x$-axis. (TDC) denotes the run in which a different TDC clock was used. Runs in the magnetic field of $118 \mathrm{mT}$ have larger errors because the runs are performed for shorter periods.

\begin{tabular}{ccccc} 
Run & Magnetic Field $(\mathrm{mT})$ & $\Omega(\mathrm{rad} / \mathrm{ns})$ & HFS $(\mathrm{GHz})$ & Events \\
\hline 100mT (down) & 100.592 & $0.24588 \pm 0.00015$ & $203.04 \pm 0.12$ & $2.7 \times 10^{8}$ \\
100mT (up) & 100.594 & $0.24573 \pm 0.00017$ & $203.17 \pm 0.14$ & $2.1 \times 10^{8}$ \\
$118 \mathrm{mT}$ (down) & 118.824 & $0.34242 \pm 0.00032$ & $203.42 \pm 0.19$ & $4.9 \times 10^{7}$ \\
$118 \mathrm{mT}$ (up) & 118.826 & $0.34289 \pm 0.00036$ & $203.14 \pm 0.21$ & $6.5 \times 10^{7}$ \\
$118 \mathrm{mT}$ (up,TDC) & 118.826 & $0.34207 \pm 0.00039$ & $203.63 \pm 0.23$ & $6.5 \times 10^{7}$ \\
$135 \mathrm{mT}$ (down) & 134.805 & $0.44034 \pm 0.00025$ & $203.58 \pm 0.11$ & $1.9 \times 10^{8}$ \\
$135 \mathrm{mT}$ (up) & 134.807 & $0.44104 \pm 0.00030$ & $203.26 \pm 0.14$ & $2.1 \times 10^{8}$ \\
$138 \mathrm{mT}$ (down) & 138.326 & $0.46394 \pm 0.00031$ & $203.45 \pm 0.14$ & $9.7 \times 10^{7}$ \\
$138 \mathrm{mT}$ (up) & 138.330 & $0.46414 \pm 0.00027$ & $203.37 \pm 0.12$ & $2.0 \times 10^{8}$
\end{tabular}

Table 4: Summary of the HFS values obtained using the subtracting method. (up) and (down) denote the direction of the $\beta^{+}$with respect to the $x$-axis. (TDC) denotes the run in which a different TDC clock was used. Runs in the magnetic field of $118 \mathrm{mT}$ have larger errors because the runs are performed for shorter periods.

\begin{tabular}{ccccc} 
Run & Magnetic Field $(\mathrm{mT})$ & $\Omega(\mathrm{rad} / \mathrm{ns})$ & HFS $(\mathrm{GHz})$ & Events \\
\hline 100mT (down) & 100.592 & $0.24579 \pm 0.00012$ & $203.11 \pm 0.10$ & $2.7 \times 10^{8}$ \\
100mT (up) & 100.594 & $0.24558 \pm 0.00014$ & $203.30 \pm 0.12$ & $2.1 \times 10^{8}$ \\
$118 \mathrm{mT}$ (down) & 118.824 & $0.34248 \pm 0.00027$ & $203.38 \pm 0.16$ & $4.9 \times 10^{7}$ \\
$118 \mathrm{mT}$ (up) & 118.826 & $0.34289 \pm 0.00028$ & $203.15 \pm 0.17$ & $6.5 \times 10^{7}$ \\
$118 \mathrm{mT}$ (up,TDC) & 118.826 & $0.34221 \pm 0.00031$ & $203.55 \pm 0.18$ & $6.5 \times 10^{7}$ \\
$135 \mathrm{mT}$ (down) & 134.805 & $0.44061 \pm 0.00020$ & $203.46 \pm 0.09$ & $1.9 \times 10^{8}$ \\
135mT (up) & 134.807 & $0.44103 \pm 0.00023$ & $203.27 \pm 0.11$ & $2.1 \times 10^{8}$ \\
$138 \mathrm{mT}$ (down) & 138.326 & $0.46401 \pm 0.00024$ & $203.42 \pm 0.11$ & $9.7 \times 10^{7}$ \\
$138 \mathrm{mT}$ (up) & 138.330 & $0.46422 \pm 0.00022$ & $203.34 \pm 0.09$ & $2.0 \times 10^{8}$
\end{tabular}

Table 3: An example of the fitted result using the subtracting method for $100 \mathrm{mT}$ (up).

\begin{tabular}{cc} 
Parameter & Fitted Value \\
\hline$A$ & $0.0056 \pm 0.0042$ \\
$B$ & $-0.0015 \pm 0.0043$ \\
$C$ & $0.0377 \pm 0.0008$ \\
$D$ & $0.00013 \pm 0.00002$ \\
$\gamma_{1}\left(\mathrm{~ns}^{-1}\right)$ & $0.0077 \pm 0.0010$ \\
$\gamma_{2}\left(\mathrm{~ns}^{-1}\right)$ & $0.0094 \pm 0.0010$ \\
$\Omega(\mathrm{rad} / \mathrm{ns})$ & $0.24558 \pm 0.00014$ \\
$\theta(\mathrm{rad})$ & $0.16 \pm 0.02$ \\
\hline$\chi^{2} / \mathrm{ndf}$ & $1.00(\mathrm{ndf}=1392)$ \\
\hline
\end{tabular}

\section{Discussion and result}

\subsection{Systematic errors}

The systematic errors are summarized below:

1. Varying the frequency sweep range of the NMR magnetometer results in slightly different readings. The uncertainty in the magnetometer calibration is estimated from this deviation (35 ppm).

2. The non-uniformity of the magnetic field results in the following two effects: (1) The oscillations in the time spectra become smeared. This effect is already taken into account in the fitted results listed in Table 2 and 4. (2) There may be a difference between the value of the magnetic field strength as measured by the NMR and the actual values over the range of the aerogel. This effect is estimated at $10 \mathrm{ppm}$.

3. The accuracy of the TDC is determined by that of the clock (Hittite HMC-T2000), which is better than 2 ppm. The effects of differential and integral non-linearities in the TDC are negligible.

4. The fitting region dependence is negligible as long as the fitting start time is later than $50 \mathrm{~ns}$ for the separate fitting method, and $16 \mathrm{~ns}$ for the subtracting method.

5. In the subtracting method, remnant exponential components could affect the HFS value in fitting procedures. This amount is estimated by altering the scale factor for each histogram by $5 \%$, which result in the shift of $10 \mathrm{ppm}$ in the HFS value. In the subtracting method, weighted average of the remnant exponential components over all runs is only $0.7 \%$, which causes a negligible effect on the HFS value.

6. An electric field produced by aerogel grains varies the overlap of the wave function of positronium, which results in a shift in the HFS (Stark effect). Since a hydrophobic silica aerogel (hydroxyl groups are replaced with trimethyl-silyl groups) is used in this experiment, the effect of Stark shift is rather small. The amount of Stark effect is estimated as follow [15]:

The area density of silanol groups of the aerogel $(\sigma=$ $0.44 \mathrm{~nm}^{-2}$ ) is measured in Ref. [15]. Although the density of aerogel is different, the primary grain in the both aerogels is the same. Furthermore, the same chemical processes to replace silanol group is applied. The average 
electric field which affects positronium during its flight can be calculated,

$$
\begin{aligned}
\overline{|E|^{2}} & \simeq\left(\frac{1}{4 \pi \epsilon_{0}}\right)^{2} \frac{\pi \sigma p^{2}}{2 \bar{L} a_{\mathrm{Ps}}^{3}} \\
& =1.0 \times 10^{16} \mathrm{~V}^{2} / \mathrm{m}^{2},
\end{aligned}
$$

where $p=1.7 \times 10^{-18} \mathrm{esu} \cdot \mathrm{cm}$ is electric dipole moment of hydroxyl groups [16], $\bar{L}=130 \mathrm{~nm}$ is the mean distance between the grains, and $a_{\mathrm{Ps}}=0.106 \mathrm{~nm}$ is the Bohr radius of positronium. The amount of the shift in the HFS is then [17]

$$
\begin{aligned}
\frac{\delta \text { HFS }}{\text { HFS }} & =-248 \cdot \frac{\overline{|E|^{2}}}{E_{0}^{2}} \\
& =-10 \mathrm{ppm},
\end{aligned}
$$

where $E_{0}=m_{\mathrm{e}}^{2} e^{5} / \hbar^{4}=5.14 \times 10^{9} \mathrm{~V} / \mathrm{cm}$. This model reproduces the experimental values in Ref. [18] within a factor 2. This Stark effect is treated as the systematic error in this analysis.

Since $\Delta_{\text {HFS }}$ depends on the magnetic field squared, the systematic errors due to the magnetic field uncertainty are doubled. The systematic errors are combined by summing in quadrature.

\subsection{Discussion}

$\Delta_{\mathrm{HFS}}=$ $203.336 \pm 0.048$ (stat.) \pm 0.015 (sys.) GHz (separate fitting) $203.324 \pm 0.039$ (stat.) \pm 0.015 (sys.) GHz (subtracting)

We note that the results of the two different fitting methods are consistent. We use the more accurate subtracting method value as the final result. The accuracy is $200 \mathrm{ppm}$, which is an improvement by a factor 90 over the previous experiment which used the oscillation method [12]. This result is consistent with both the theoretical calculation [1] and the previous more precise experimental values which directly measure the Zeeman transition [2, 3].

In order to observe the relaxation of positronium spin (Ps$\mathrm{SR}$ ), the oscillation amplitude is fitted as a function of time. The result is, however, consistent with a constant. A better accuracy and a higher density target are necessary to observe the relaxation.

The accuracy of the measured HFS value in this study is $200 \mathrm{ppm}$. The following five points can be improved in order to achieve a better accuracy:

1. Increase the total run time by a factor 20 (about 1.5 years).

2. Increase the intensity of the radioactive source by a factor 3. The dead time of the DAQ would still be acceptable.

3. Increase the coverage of the photon detectors by a factor 3. Fine segmentation is still necessary.

4. The absolute calibration of the NMR and the uniformity of the magnetic field can both easily be improved to $O(1) \mathrm{ppm}$.

5. The Stark effect can be estimated by changing the density of the aerogel.
The result of these improvements would be an increase in statistics by a factor 180 and an improvement of the final accuracy to about $15 \mathrm{ppm}$.

\section{Acknowledgements}

We thank Dr. M. Ikeno (KEK) for the development of the TDC. Sincere gratitude is also expressed to Mr. M. M. Hashimoto and Dr. T. Tanabe for the useful discussions.

\section{References}

[1] B. A. Kniehl and A. A. Penin, Phys. Rev. Lett. 85 (2000) 5094; K. Melnikov and A. Yelkhovsky, Phys. Rev. Lett. 86 (2001) 1498; R. J. Hill, Phys. Rev. Lett. 86 (2001) 3280.

[2] A. P. Mills, Jr., and G. H. Bearman, Phys. Rev. Lett. 34 (1975) 246; A. P. Mills, Jr., Phys. Rev. A 27 (1983) 262.

[3] M. W. Ritter, P. O. Egan, V. W. Hughes, and K. A. Woodle, Phys. Rev. A 30 (1984) 1331.

[4] G. W. Bennett et al., Phys. Rev. D 73 (2006) 72003.

[5] A. Ishida et al., arXiv:1004.5555 (2010).

[6] S. Asai, S. Orito, and N. Shinohara, Phys. Lett. B. 357 (1995) 475; R. S. Vallery, P. W. Zitzewitz and D. W. Gidley, Phys. Rev. Lett. 90 (2002) 203402.

[7] V. G. Baryshevsky, O. N. Metelitsa, and V. V. Tikhomirov, J. Phys. B 22 (1989) 2835.

[8] H. Grotch and R. A. Hegstrom, Phys. Rev. A 4 (1971) 59; E. R. Carlson, V. W. Hughes, M. L. Lewis, and I. Lindgren, Phys. Rev. Lett. 29 (1972) 1059; H. Grotch and R. Kashuba, Phys. Rev. A 7 (1973) 78; M. L. Lewis and V. W. Hughes, Phys. Rev. A 8 (1973) 625.

[9] New experimental approach without Zeeman effect has been proposed in S. Asai et al., arXiv:1003.4324 (2010). The direct transition from $o$-Ps to p-Ps is made using the high power sub-THz light source.

[10] M. Deutsch and S. C. Brown, Phys. Rev. 85 (1952) 1047; V. B. Berestetskii and L. D. Landau, J. Exptl. Theoret. Phys. (U.S.S.R.) 19 (1949) 673 , 1130; O. Halpern, Phys. Rev. 94 (1954) 904.

[11] V. G. Baryshevsky, O. N. Metelitsa, V. V. Tikhomirov, S. K. Andrukhovich, A. V. Berestov, B. A. Martsinkevich, and E. A. Rudak, Phys. Lett. A 136 (1989) 428.

[12] S. Fan, C. D. Beling, and S. Fung, Phys. Lett. A 216 (1996) 129.

[13] E. Ivanov, I. Vata, S. Teodorian, I. Rusen, and N. Stefan, Nucl. Inst. Meth. B 267 (2009) 347.

[14] Lifetime becomes shorter due to the pick-off collision with the aerogel. Y. Kataoka, S. Asai, and T. Kobayashi, Phys. Lett. B. 671 (2009) 219; O. Jinnouchi, S. Asai, and T. Kobayashi, Phys. Lett. B. 572 (2003) 117.

[15] Y. Kataoka, Doctoral thesis, 2007, University of Tokyo.

[16] E. M. Purcell, Electricity and Magnetism, 2nd edition Berkeley Physics Course. Volume 2, McGraw-Hill, Inc. New York.

[17] G. W. Ford, L. M. Sander, and T. A. Witten, Phys. Rev. Lett. 36 (1976) 1269.

[18] M. H. Yam, P. O. Egan, W. E. Frieze, and V. W. Hughes, Phys. Rev. A 18 (1978) 350. 